\title{
The Control of Body Position in the Stick Insect (Carausius morosus), when Walking over Uneven Surfaces ${ }^{\star}$
}

\author{
H. Cruse \\ Fachbereich Biologie der Universität, Kaiserslautern, FRG
}

\begin{abstract}
An investigation has been made of the way, in which the height of the body of an insect (Carausius morosus) is controlled when walking over an uneven terrain. The animals have been filmed from the side while walking over different types of irregularity (step up, step down, obstacle, ditch). A frame by frame analysis of the height of the three thoracic segments of the insect has been performed. A computer model has been set up, which is able to describe the experimental results within the exactness of measurement. This model consists of three independent height controllers, each having a unique characteristic. The coupling of these three controllers is performed mechanically. One possible interpretation of this model is that the height of each segment is controlled by a closed loop mechanism with a proportional element as a controller.
\end{abstract}

\section{A. Introduction}

Until now there exist very few experimental results dealing with the control of body position in free walking insects. Recent investigations describe only menotactic control of the longitudinal axis of the body relative to directed stimuli, e.g. gravity or light (Bässler, 1975; see here for further references, Wendler, 1972). The present paper describes the manner in which the body position is influenced by the shape of the walking surface. This problem has only been investigated up to now in an indirect way. For example Bässler (1965, 1967, 1972a, b, 1973, 1974) and Wendler $(1964,1972)$ have examined the mechanisms, that control the angle of leg joints in standing or fixed animals. Although it may be of great practical interest for the construction of walking machines able to travel over an irregular terrain, one has no idea about the mechanisms which

* Supported by the Deutsche Forschungsgemeinschaft a six-legged animal applies when walking over such a surface. Only Okhotsimskiy et al. (1972) have constructed a mathematical model describing a six-legged apparatus able to run over uneven terrain, by using data which have been obtained by measuring the coordination of the legs of an insect (Wilson, 1966). The decisive disadvantage of Okhotsimskiy's model is, that it only works, if the shape of the walking surface does not deviate from a given horizontal line by more than in a defined amount. This depends on the length of the leg-limbs. As moreover the data of Wilson are only concerned with the coordination of the six legs, the present investigation investigates the way, in which a free walking insect changes its body position when walking over several differently shaped obstacles. From the preliminary results a model is formulated to describe the body-movements of the animal under the investigated conditions.

\section{B. Method}

The animals used in this investigation were adult stick insects (Carausius morosus, Phasmids) reared at this institute. In order to be able to evaluate the height of the different parts of the body of the walking insect, the animals have been filmed with a super 8 camera (18 frames/s) from the side, when walking over a horizontal plane. This walking surface (length $55 \mathrm{~cm}$, breadth $14 \mathrm{~cm}$ ) was made of styrofoam. Later experiments have been performed using a mirror at $45^{\circ}$ to the walking plane, so that the animal could be seen simultaneously in plane and elevation. In the middle of the walking plane, perpendicular to the walking direction, different types of irregularities were constructed: either an obstacle (height $6 \mathrm{~mm}$, breadth $10 \mathrm{~mm}$ ), or a ditch (depth $10 \mathrm{~mm}$, breadth, $15 \mathrm{~mm}$ ) or steps of different height $(7 \mathrm{~mm}, 8.6 \mathrm{~mm}, 14 \mathrm{~mm})$. Concerning the latter one must discriminate between "up the step" and "down the step" walks.

To measure the height of the body of the insect when walking over the plane and the different irregularities, the films were evaluated frame by frame with the aid of a single frame projector and a digitizer combined with a programmable calculator (Hewlett-Packard 9810). In each frame the three distances from the fore-, middle-, and hind coxae to the baseline, given by the level of the styrofoam plane, were computed. Figure 1 shows these distances 


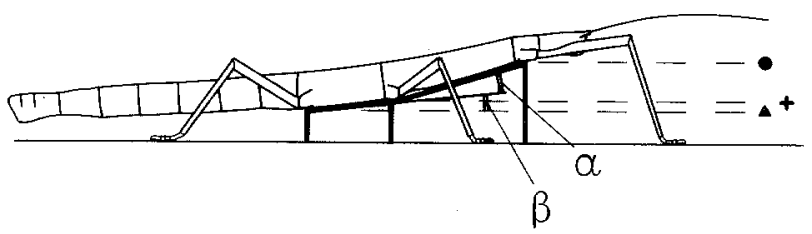

Fig. 1. Schematic presentation of the measured values; $(\bullet)$ height of the prothoracic coxae, $(+)$ height of the mesothoracic coxae, $(\boldsymbol{\Lambda})$ height of the metathoracic coxae, $\alpha$ angle between the meso- and metathorax, $\beta$ angle between the metathorax and the horizontal baseline

as well as the two angles $\alpha$ and $\beta$, which additionally were computed by the calculator. Angle $\alpha$ is a measure of the angle between the mesothorax and the metathorax, while $\beta$ describes the angle between the metathorax and the horizontal baseline. The height of the coxae of the three thoracic segments computed with respect to the baseline are symbolized in Figure 1 as well as in the figures to come in the following way: the height of the coxae of the prothorax by a circle, that of the mesothorax by a cross and that of the metathorax by a triangle. In the case of the step-shaped irregularities one can think of two different baselines, an upper and a lower one. To make the measurements comparable in these cases the lower baseline was always used as a reference line. The total number of animals used in these experiments was 18 , the total number of runs analysed was 60 . The number of frames examined was 3411 .

\section{Results}

In order to measure the change in the height of the different parts of the body, walks over six distinct types of obstacles have been investigated. Figure $2 \mathrm{a}$ shows the results obtained by walks over an upward step (height $8.6 \mathrm{~mm}$ ). In order to average the data over different runs and animals different configurations of the animal's legs relative to the obstacle are defined. For all values of one type of configuration the mean values and the standard deviations of the height of the fore $(\bullet)$, the middle $(+)$ and the hind coxae $(\boldsymbol{\Lambda})$ were computed and plotted in Figure 2. The different configurations are defined by the arrangement of the six tarsi relative to the obstacle. They are symbolized in Figure 2 on the abscissa. The baseline is always represented as a dashed line. The first configuration describes the animal approaching the step. In the second configuration one tarsus of the fore legs touches the upper level of the step. In the third configuration both tarsi of the fore legs touch the upper level of the step. In the following configurations successively the remaining tarsi walk on the step, until in the last configuration all six legs of the animal have reached the upper level of the step. When a leg is in the protraction movement, these frames are treated as belonging to the earlier configuration as long until the tarsus of this leg touches the ground. Then depending on the locus at which the tarsus is touching the ground, the animal can be regarded as being in
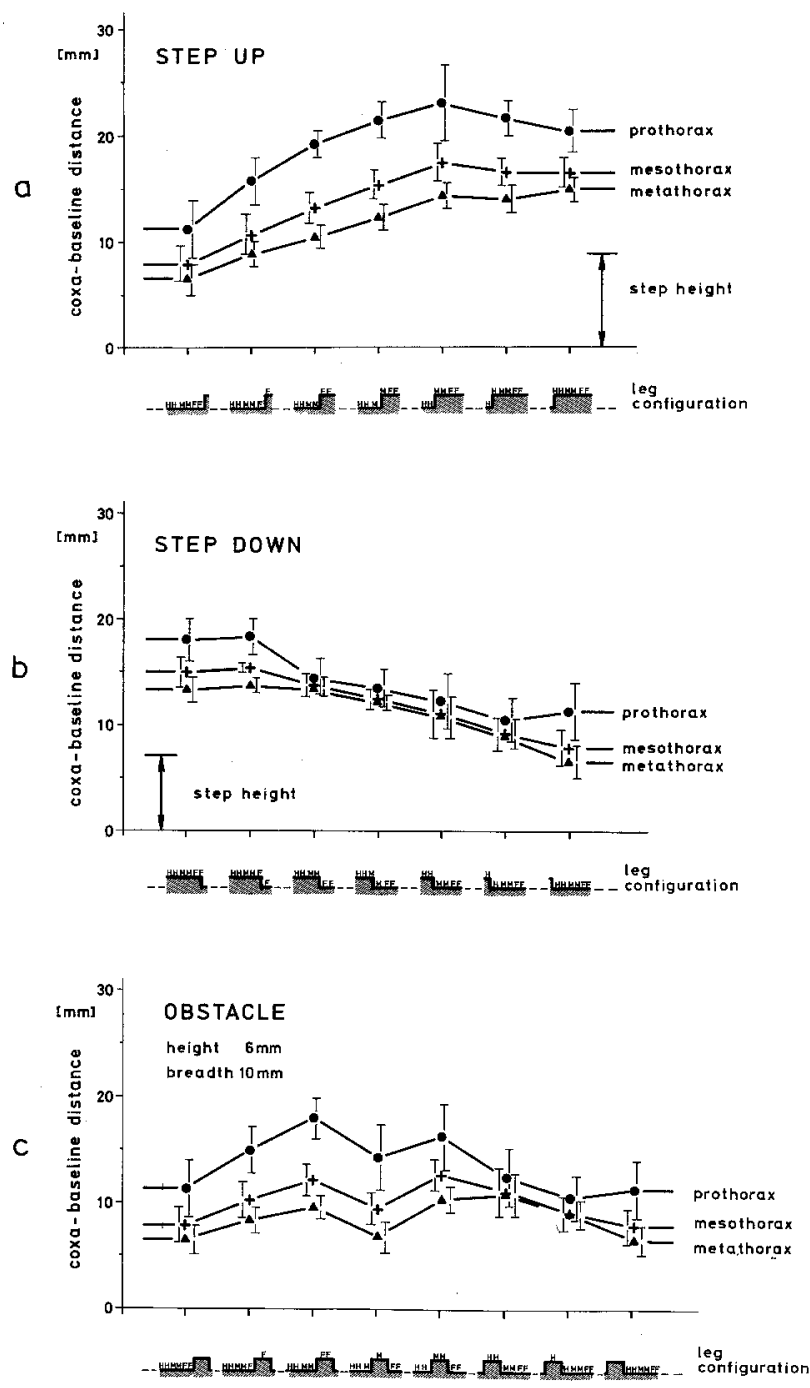

Fig. 2a-c. The heights of the coxae of the three thoracical segments are shown, when the insect walks over (a) a "step up", (b) a "step down" and (c) an "obstacle". The values are given for different types of leg configurations as shown on the abscissa. The bars show the values of the standard deviations of the corresponding mean values. For further explanation see the text

the next configuration. While in one single walk for the first and the last step arrangement (when the animal walks on the horizontal plane) you get up to 60 frames, for the remaining step arrangements this number is much lower (2-10).

As you can see in Figure 2a, in all configurations the coxae of the prothorax are higher than those of the mesothorax, and the latter again are higher than those of the metathorax. This means, that the angles $\alpha$ and $\beta$ always have positive values. Another point is, that the coxae of the metathorax begin to be lifted in the first four configurations, although in these positions the tarsi of both hind legs have not yet walked over the step. A third observation is, that the 
standard deviations of the values of the meso- and metathorax are lower than those of the prothorax. Particularly this is true for the first and the last configuration, where the animal walks over the horizontal plane. This certainly depends on the fact, that stick insects, walking on a horizontal plane, very often make searching movements with their fore legs, in horizontal as well as in vertical directions. Therefore the height of the prothorax changes much more than that of the meso- and metathorax.

In the same way Figure $2 b$ shows the results, when the animals walk over a step downward (height $7 \mathrm{~mm}$ ). Again the coxae of the prothorax lie higher than those of the meso- and metathorax, but the distance is smaller. Between the third and the sixth configuration the coxae of meso- and metathorax have about the same height. This means, that the angle $\beta$ is approximately zero, while $\alpha$ is still positive. It is of interest, that in the second step arrangement the body position shows no change, although the tarsus of one fore leg touches the lower ground.

Figure $2 \mathrm{c}$ shows the results of animals walking over a rectangular obstacle with a height of $6 \mathrm{~mm}$ and a breadth of $10 \mathrm{~mm}$. The series of positions on the abscissa does not necessarily show the real temporal order. For to give an extreme example, you sometimes find a walk with all legs striding across the obstacle in such a way, that no tarsus touches it at all. The first three and the last two configurations correspond to those appearing when the animal walks over "a step up" and "a step down" and so do the results. Only the fourth and the fifth configurations are novel. Although in these two configurations only the tarsi of the mesothorax stand on the obstacle, the arrangement of the symbols describing the height of the three segments does not essentially differ from that in the horizontal plane situation.

In consequence of these first experimental results a model was built up describing the different body positions of the insect when walking over the step up, the step down and the obstacle. To compare the model behaviour (which is described in Section E), with that of the animals three additional experiments have been done. Figure $3 a$ shows the experimental results, when the insects walk over a ditch of a depth of $10 \mathrm{~mm}$ and a breadth of $15 \mathrm{~mm}$. As in the case of the obstacle and for the same reason the positions on the abscissa do not necessarily give the real temporal order. Often some of these step arrangements have been omitted and sometimes other step arrangements could be found, the number of which however was too small to compute significant mean values and standard deviations.

The main difference between the results shown in Figure $3 \mathrm{a}$ and the earlier results is, that in Figure $3 \mathrm{a}$ in
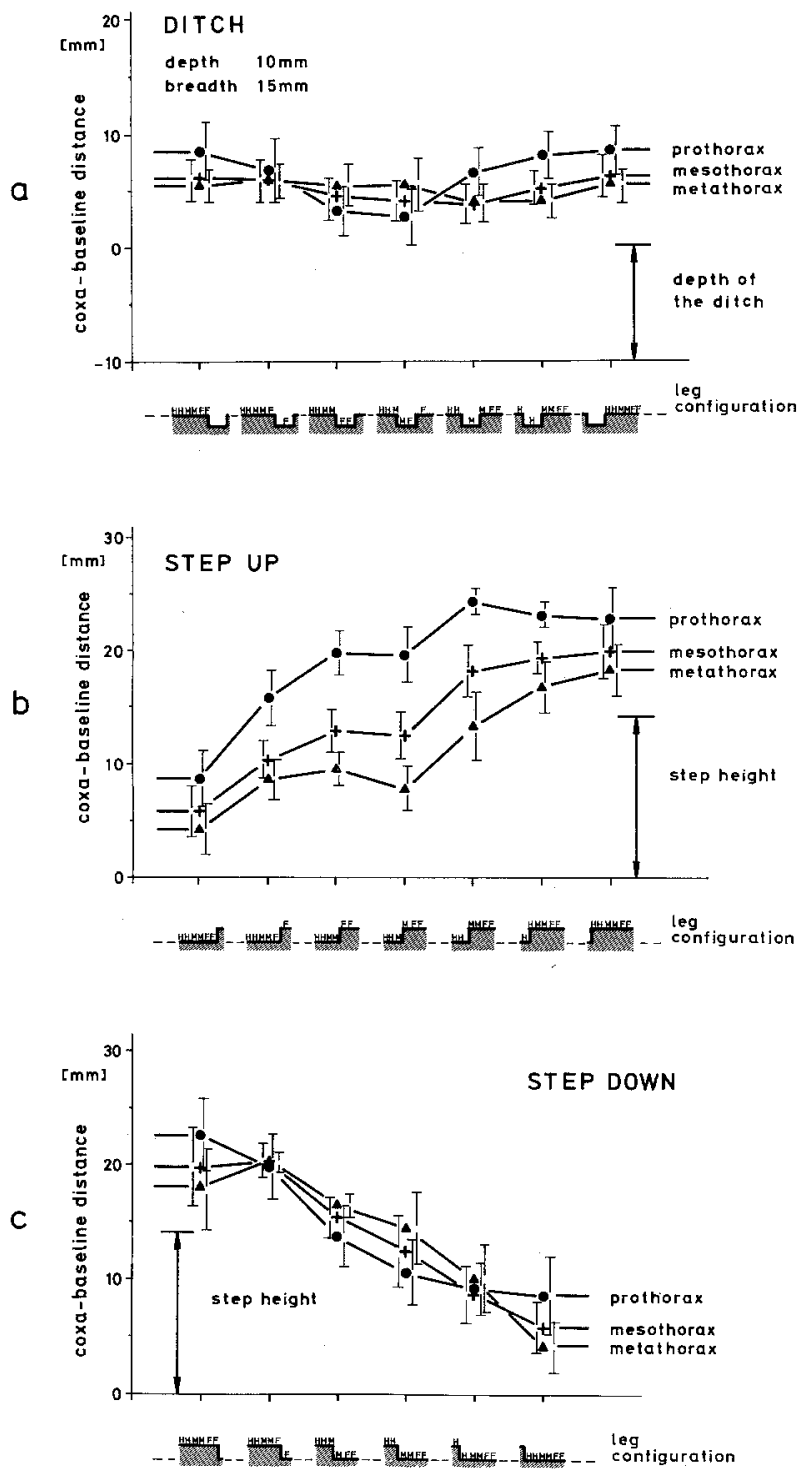

Fig. 3a-c. The heights of the coxae of the three thoracical segments are shown, when the insect walks over (a) a "ditch", (b) a "step up" and (c) a "step down". The values are given for different types of leg configurations as shown on the abscissa. The bars show the values of the standard deviations of the corresponding mean values. For further explanation see the text

the third and the fourth configuration the coxae of the prothorax lie below those of the mesothorax and these again are below those of the metathorax. As the third configuration also occurs when walking down a step, this effect is probably produced by the increased depth of this "step". Thus the angle $\beta$ in these two configurations becomes negative, while angle $\alpha$ still has a positive value. This can be estimated from the fact, that the distances between the heights of the coxae of pro- and mesothorax as well as of meso- and metathorax are nearly equal (Fig. 3a, configuration 3, 4), while the distance between the coxae of the meso- and 
metathorax is smaller than that between the coxae of the pro- and the mesothorax (Fig. 1).

The last two experiments have been done with steps up and down as above but with a much greater height $(14 \mathrm{~mm})$. This is a considerable irregularity for the stick insects used here as one can see, when comparing the height of the step with that of the body in normal horizontal walking (Fig. 3b, c). As in Figure 3b (step up) no qualitative difference relative to Figure $2 \mathrm{a}$ can be seen, but Figure $3 \mathrm{c}$ shows a significant difference compared to Figure 2b. Here, as in Figure 3a, in some configurations the order of the height of the coxae is the inverse of the normal order. As no parameter has been changed except the height, it can be assumed, that the increase in step height alone is responsible for the inversion in the order of the segmental heights. The standard deviations of the results shown in Figure $3 \mathrm{~b}$ and $\mathrm{c}$ are greater than those in the other experiments. This is to be expected because of the greater height of the irregularities, which cause the body to move through a greater distance in the same time interval, resulting in a greater standard deviation. Therefore in these cases the standard deviation is not a very good measure for the description of the exactness of the mean values.

\section{The Angle between Meso- and Metathorax}

To construct a six-legged apparatus able to walk over an uneven terrain, among others one can think of two possible models. One model, which corresponds to that described by Okhotsimskiy et al. (1972), regards the body of the animal as being rigid with the longitudinal axis of the body always being in a horizontal position. At the end of the protraction movement of one leg in this model the tarsus moves down until it touches the ground. The height of the coxa of this leg will then be held constant during the retraction movement of the leg. This model in rather a simple way enables the body to hold a constant position of the longitudinal axis of the body relative to a horizontal line independent of the shape of the walking surface. On the other hand it has the disadvantage, that, when walking from a horizontal plane onto one with an upward slope, the distance between the body and the ground progressively decreases until it becomes zero, or, if the plane has a downward slope, this distance increases until all the legs are completely stretched. This means, that the animal then could not move forward. One way to make the model more realistic would be to add a further mechanism, which for example holds the averaged height of the six coxae constant. This mechanism would require a neuronal network with afferent and efferent channels to the legs. Therefore

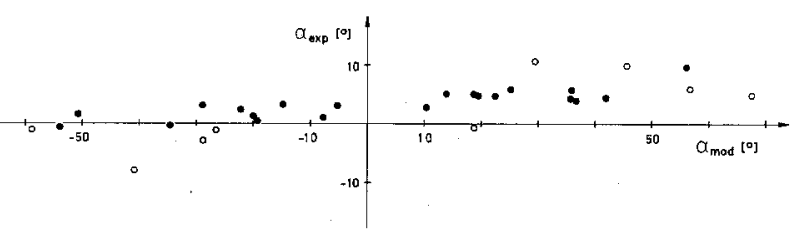

Fig. 4. The values of the measured angle $\alpha_{\exp }$ plotted against the values of $\alpha_{\text {mod }}$ which would be expected, if the three thoracical segments controlled their height completely independent from one another and the thoracical joints could be moved free. The open circles represent the values obtained by the experiments shown in Figure $3 b, c$

the "first approximation" model of Okhotsimskiy et al. becomes somewhat more complicated, if one tries to apply it directly to a real system.

Another possible model uses the assumption, that each pair of legs holds constant the individual height of its segment independent of the other legs. This model might describe the behaviour of quadrupedal mammals as it seems to you to be for example with dogs walking free over an uneven surface. In sixlegged insects this is only possible, if the thoracic joints can be moved in the walking animal within their morphologically given limits. A change of the angle between pro- and mesothorax in the stick insect does not essentially change the differences in the heights between the coxae of the prothorax and the mesothorax, because the distance between the promesothoracic joint and the coxae of the prothorax is very small (Fig. 1). Therefore in this sense only the meso-metathoracic joint seems to be important. This means, that for this model the value of the angle $\alpha$ (Fig. 1) should depend very much on the shape of the walking surface. Since in the model discussed first the angle $\alpha$ always has to be constant, the measurement of this angle offers a possibility to discriminate between these two models. Therefore for the different configurations shown in Figures 2 and 3 the expected values of $\alpha$ were computed assuming the second model to be true. These values $\alpha_{\text {mod }}$ are compared with the values $\alpha_{\text {exp }}$, which had experimentally been measured in the corresponding step arrangements by plotting $\alpha_{\text {exp }}$ against $\alpha_{\text {mod }}$ (Fig. 4). If the two legs of one segment do not stand on the same level as it is the case in some configurations, the height of the coxae of this segment would have two different values. Here it is supposed, that the mean value of both was used to describe the height of these coxae. For a better discrimination the values of the last two experiments (Fig. 3b, c) are plotted as open circles. If the experiments could be described by the second model, which would mean that $\alpha_{\bmod }$ is equal to $\alpha_{\exp }$ one would expect the points in Figure 4 to be arranged in a straight line with slope 1 . 
If on the other hand the first model fitted the data, a horizontal line would be the result. As the linear regression of these data gives the equation $\alpha_{\exp }=2.68+$ $0.082 \alpha_{\bmod }$, the value of the slope is very near to zero, even if the difference from zero is significant $(p<0.001)$. Although in consequence of this there seems to be a very small dependence of $\alpha_{\exp }$ on the shape of the walking surface, as a first approximation one can exclude the second model. So one can conclude from these results, that the stick insect holds it's body nearly completely rigid when walking over obstacles with the dimensions used in these experiments.

To test whether this is still true in more extreme cases, the animals were filmed when changing from a horizontal walk to a climb up a vertical plane. Then the angle $\alpha$ can reach maximal values between $20^{\circ}$ and $30^{\circ}$. If the body was still rigid, when changing from the horizontal to the vertical plane, the middle as well as the hind legs wouldn't be able to touch the ground. In order to do this the body has to be bent at the mesometathoracic and metathorax-abdominal joints. When on the contrary the insect had to walk from a horizontal plane over an edge downwards on a vertical plane, one could never find values of $\alpha$ which were significantly lower than zero. The animals in this case never.walk perpendicular to the edge, but always turn about $90^{\circ}$ so that the longitudinal axis of the body is nearly parallel to the edge. This is also frequently true in the first situation, when climbing up the vertical plane.

These results show, that holding the body rigid when walking over the less extreme irregularities is not a consequence of a morphological limitation of the joints but is an active process controlled by the muscles of the intersegmental joints. To determine the range of the angle $\alpha$ the following experiment has been done. The insects were glued at the dorsal part of the metathorax and then with a horizontal position of the metathorax were held free in the air. Then the value of the angle between meso- and metathorax was measured by a shadow projection of the body during active searching movements of the animal as well as during a careful bending of the body by hand. The extreme values of the angle $\alpha$ when bent by hand were $\alpha_{\max }=40^{\circ}$ and $\alpha_{\min }=-40^{\circ}$, and in active movements they were $\alpha_{\max }=30^{\circ}$ and $\alpha_{\min }=-20^{\circ}$. This shows clearly, that the small range of $\alpha$ values observed when the animal walks across the surfaces described above (Fig. 2,3) are not due to morphological limitations of the joint.

\section{E. Model}

Except of these extreme situations just discussed one can say as a first approximation, that when walking over irregularities as investigated here, the body of the insect can be regarded as rigid, and therefore the second model described in Section $\mathrm{D}$ can be excluded. Although the rigidity of the body agrees with the first model described in Section D, you must also exclude the first model because of other properties of the experimental results. So the position of the body described by the angle between the metathorax and the horizontal baseline (Fig. 1, angle $\beta$ ) is not always constant as required by the first model. As a rough approximation this angle $\beta$ corresponds to the mean slope of that part of the walking surface, which is situated within the range of the animal's body. This means, that if the animal climbs up, as in the step up or the first part of the obstacle or the second part of the ditch, the angle $\beta$ is greater, and when climbing down, $\beta$ is smaller compared withe the value of $\beta$ when the animal walks on the horizontal surface. While, when walking over the horizontal plane, the mean value of the angle $\beta$ is $7.6^{\circ}\left( \pm 4.6^{\circ}\right)$, the extreme cases of the mean values in the different configurations are $\beta=31.6^{\circ}\left( \pm 7.7^{\circ}\right)$ (Fig. 3b, fifth configuration) and $\beta=-12.6^{\circ}\left( \pm 5.0^{\circ}\right)$ (Fig. 3c, fourth configuration). (The values in brackets are the standard deviations.) Therefore one could suppose, that the legs of the single segments always try to hold constant the normal height of its segment as it was postulated in the second model (Section D). Because of the rigidity of the body for geometrical reasons this is only possible when the animal walks on a flat surface without irregularities. On the other hand, when the animal walks on an uneven surface, always deviations from these normal heights are necessary. When the animal walks on a flat surface, the height of the body is the result of the effect of three different torques each of them produced by one pair of legs about a horizontal axis, which is perpendicular to the longitudinal axis of the body and which goes through its inertial centre. These torques act against one another in such a way, that the torque produced in one segment has to resist the sum of those torques, which are produced by the legs of the other two segments. The position of the body is given by the condition, that the sum of these three torques is zero.

When because of the rigidity of the body and because of an uneven surface a deviation from the normal height arises, one could put forward the hypothesis, that the torque produced by the legs of this segment is changed in dependence of the amount $\Delta h$ of the deviation. Consequently a new equilibrium state will exist which corresponds to a different position of the body. The amount of the change of the torque, which is given by a definite deviation $\Delta h$ of the actual height of the coxae from their normal height, could be described by a characteristic belonging to this seg- 


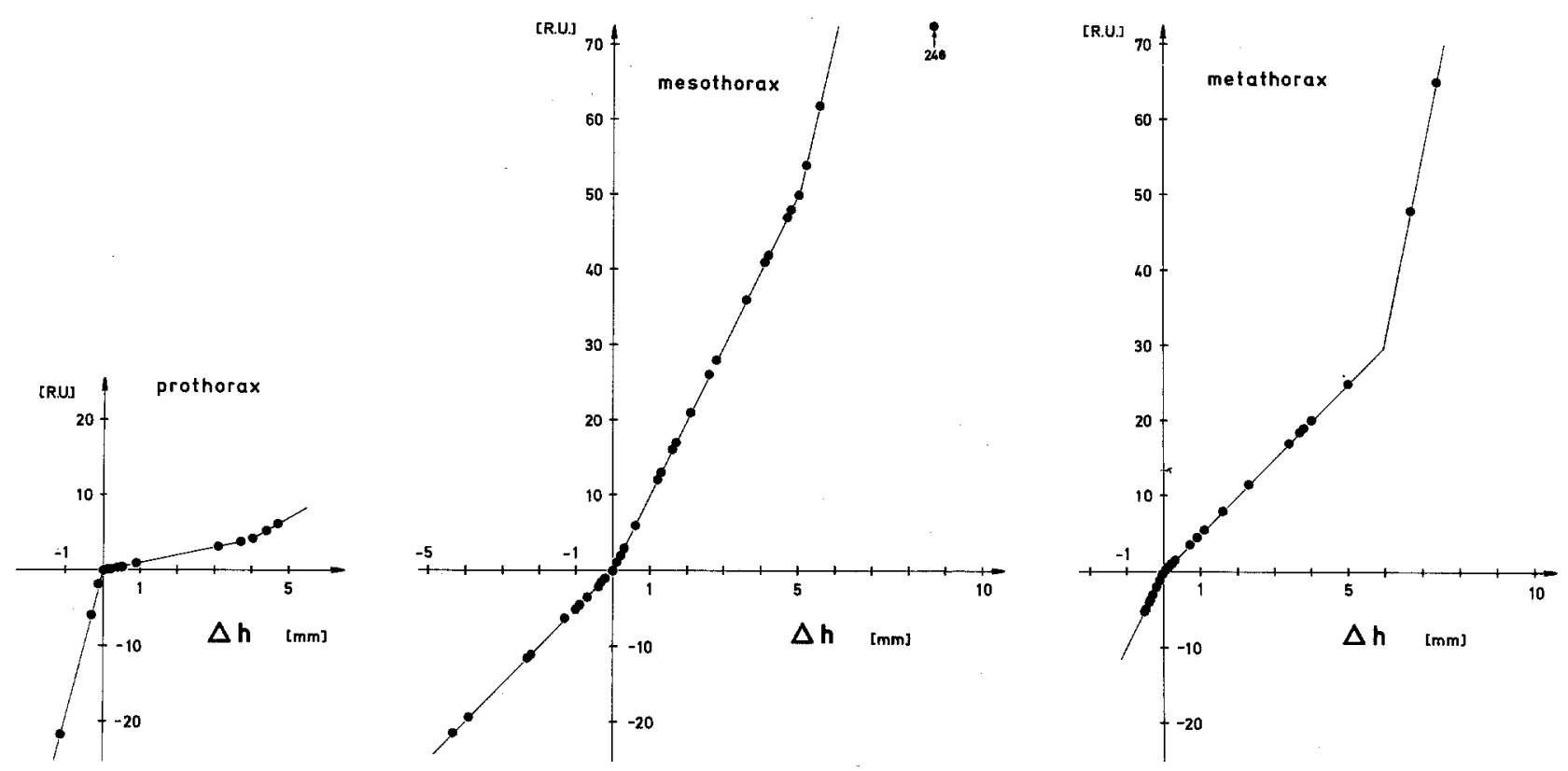

Fig. 5. The characteristics used in the model to control the height of the three thoracic segments. Each characteristic shows the relative magnitude of the change of the reaction torque of the leg pair (referred to the centre of inertia of the body) versus the deviation from the normal height. This deviation is measured as the difference $\Delta h$ between the normal height of this segment (when the insect walks over a flat horizontal surface) and its actual height. Deviations upwards are signed positive. The points are the numerical values used in the model

ment. The average force, which is produced by the legs of one segment to hold it's normal height, when the animal walks over a horizontal plane, can be called "normal force". Then these characteristics determine, apart from the effect of the lever arms, the amount of that force, about which the "normal force" is altered, when a deviation $\Delta h$ from the normal height exists. With this conception the posture of the body is given as the equilibrium state caused by the effect of the three characteristics of the segments. The posture of the body could be computed for each shape of the walking surface, if the normal height and the characteristic for each segment were known.

The following model has been computed in order to test this hypothesis and, if it would be able to describe the experimental results, to calculate the form of the characteristics of the three segments. The normal heights of the coxae of each segment are defined as the mean values of the heights of the coxae, when the insect walks on a flat horizontal surface. When the insect walks over an uneven surface, the normal height of the coxae of an individual segment relative to the baseline has been calculated in the following way. The normal height of this segment, when the animal walks over a horizontal surface has been summed with the height of the surface relative to the baseline at that point, on which both tarsi of the segment were standing. If both tarsi of one segment were standing at different heights, the mean value of these two heights was used to calculate the normal height. The angle $\alpha$ was assumed to have always the constant value of $\alpha=4.6^{\circ}$, which in the first three experiments (Fig. 2) was the mean value, when the insect walked on the horizontal surface. Then for the three segments three arbitrary characteristics have been assumed first. As the posture of the body should be the result of an equilibrium state, that posture with the smallest sum of deviations has been computed. This sum of deviations is calculated by summing up the absolute values of the three deviations $\Delta h$, weighted by the corresponding characteristic. These weighted deviations can be regarded as described above as a measure for the additional torque, which each segment produces to reach its normal height. They can be given in relative units. Since not every position of the body is possible because of the rather long abdomen (Fig. 1), additionally some mechanical limitations had to be respected in the calculation. The height of the coxae of the mesothorax was not allowed to be smaller than half height of the coxae of the prothorax, as the coxae of the metathorax lie almost exactly in the middle of the body. When walking over the ditch, the height of the coxae of the metathorax were not allowed to be smaller than the height of the walking surface. This model can then be formulated as a computer programm. A limitation 

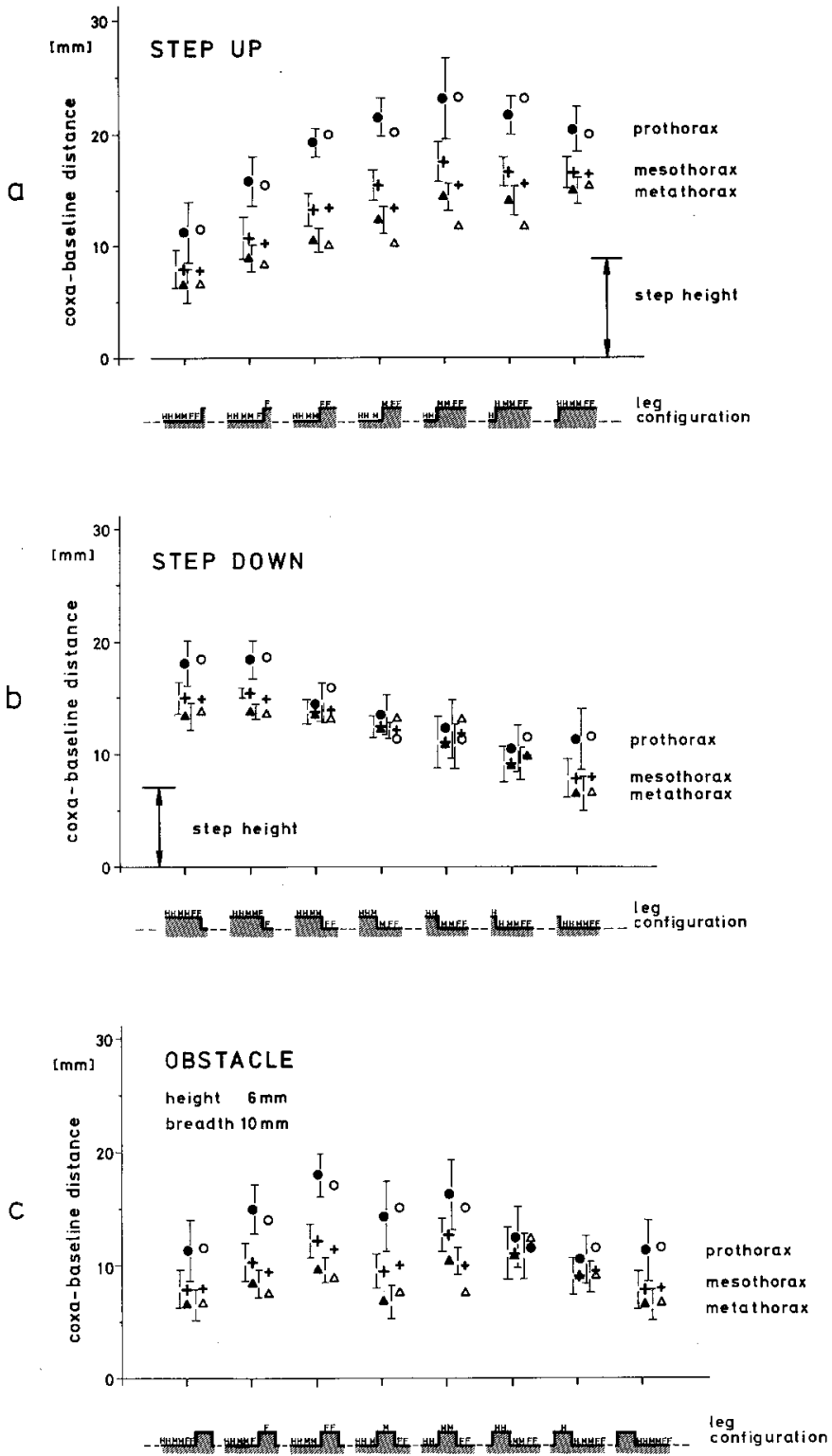
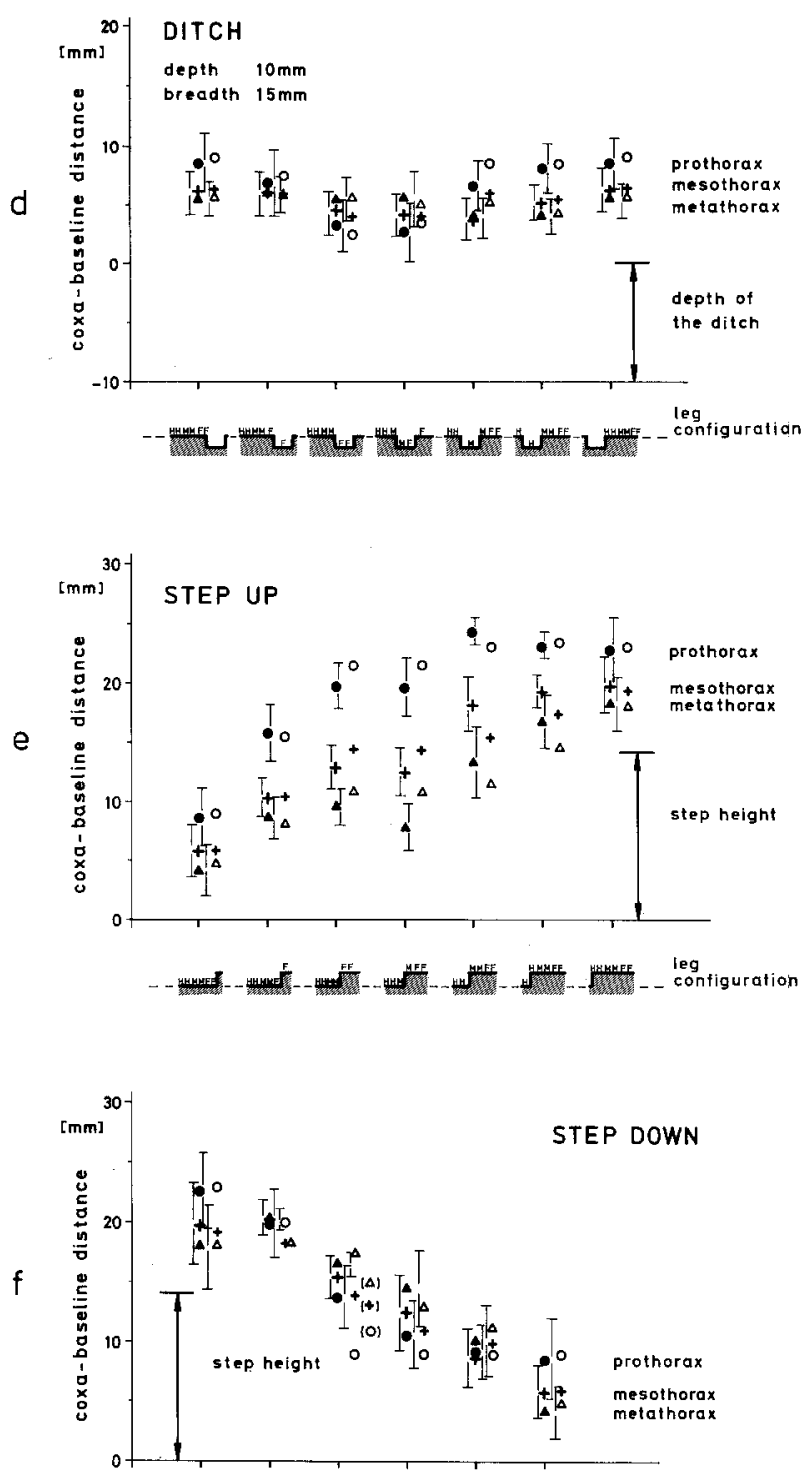

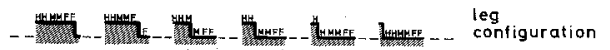

Fig. 6. Comparison of the measured values already demonstrated in Figures 2 and 3 with the corresponding values calculated by aid of the computer model. The values of the model are shown as open circles (prothorax), small crosses (mesothorax) and open triangles (metathorax)

caused by the programm was that in this calculation the values of the height of the prothorax as well as of the mesothorax could only assume discrete values in increments of $0.5 \mathrm{~mm}$. Using this programm it was possible to construct the required characteristic for each segment and obtain a good fit to the data for experiments shown in Figure 2. To provide an objective test of this model, three more experiments with different kinds of irregularities were performed, the results of which are shown in Figure 3. In order to describe all six experiments by the same characteristics, their form except of small changes in slope had only to be changed in such a way, that the range of the possible deviations had to be increased because of the higher obstacles in these experiments. The form of the characteristics used for this model for the deviation from the normal height of pro-, meso-, and metathorax are shown in Figure 5. The abscissae show the deviation $\Delta h$ of the coxae from the normal height in millimeters (deviation upwards is shown positive), while the ordinates in relative units represent the torque with which the segment acts against this forced deviation. To avoid a possible misunderstanding, it should be mentioned here, that negative values in these characteristics mean, that the value of the force, by which the corresponding legs lift up the body, is 
diminished by a definite amount. This however has not to mean, that the sign of the force vector is changed and therefore these legs try to pull the body down to the ground. In order to show the valid range of these characteristics, those values, which have been used in the calculation, are represented by points. The higher the slope of the characteristic, the more difficult is a deviation from the normal height, which here corresponds to zero. Therefore because of the very high slope of the characteristic for negative (downward) deviations in the prothorax in this case only small deviations exist. In all three segments in this model the maximal downward deviations are smaller than the maximal upward deviations. The most extreme values in both directions can be seen in the mesothorax.

To compare the properties of the model with the behaviour of the animal, the experimental values shown in Figures 2 and 3 are plotted together with the values calculated by the model in Figure 6 for the different kinds of irregularities. The experimental values are again shown as full circles, crosses and full triangles together with their standard deviations, while the model values are shown as open circles, small crosses and open triangles. As can be seen in these figures, the model discussed here as a first approximation gives a satisfying description of the experimental results. This can also be shown when comparing the mean standard deviation of all experimental results of $\bar{s}= \pm 2.18 \mathrm{~mm}$ with the standard deviation of the differences between the model values and the corresponding experimental values of $s= \pm 1.26 \mathrm{~mm}$.

When climbing down, sometimes the prothorax lies below the mesothorax. In some of these cases the model seems to show a systematic deviation from the experimental results (Fig. 6b, fourth and fifth configuration, Fig. 6f, third configuration). In this case the slope of the longitudinal axis of the body is smaller than in the model. This might depend on the fact, that stick insects as much as possible try to avoid to climb downwards. This property of these insects may be the result of an endogenous tendency to hold up the prothorax in order to reach any object to climb up. One can introduce such a tendency in the model by adding a term to the sum of the weighted deviations from the normal height. This term is proportional to the absolute value of the difference between the height of the coxae of prothorax and that of the mesothorax only if the height of the prothorax is smaller than that of the mesothorax. Otherwise it is zero. If one looks then for the characteristic of this proportionality in the same way as for the other characteristics, one finds that with this enlarged model the values of only one configuration will be changed (Fig, 6f, third configuration). These values are plotted in Figure $6 \mathrm{f}$ in brackets.

\section{F. Discussion}

The model demonstrated here represents a simple mechanism able to describe within the exactness of measurement the posture of the body of the stick insect when walking over an uneven terrain. Thereupon the question arises how this abstract model could be realized. The model says that each pair of legs of one segment tries to maintain a definite height for its segment. However, one often finds deviations from these normal heights. This is due to the fact, that the other two pairs of legs also try to keep their normal height. This condition can only be achieved for all three segments at the same time when the animal walks on a flat surface, but not when walking on an uneven surface. One interpretation of this model could be, that the heights of the different segments are controlled by feedback mechanisms, whereby the controllers act as proportional elements. The reference input of such a control mechanism is given by the normal height, the actuator by those leg muscles that are responsible for the control of the height of the segment. The influence of the other two pairs of legs could be regarded as disturbance input. The three characteristics (Fig. 5) according to this conception would be the characteristics of the three controllers including the proportional factors which represent the different moments for the single segments. This means, that these characteristics describe in a qualitative way the total effect on the change of the forces produced in vertical direction by the different muscles of the legs. The real change of the force of a single muscle also depends for example on it's geometrical situation. Therefore this change cannot be obtained from the presented characteristics without further information on the precise geometry, musculature organisation and innervation of a large number of participating muscles.

To give another interpretation of this abstract model one could assume that the heights of the coxae are not controlled by a closed loop system, but by an open loop system. This could be done by activating the supporting muscles in a "constant" manner, which brings about the normal posture of the body when walking on a flat horizontal surface. Because of the elasticity of the muscles a different posture of the body will be the result in spite of their "constant" activation (constant in a sense just described), when the animal walks over an uneven surface. Thus the three characteristics of Figure 5 might represent the elastic properties of the muscles in the state of contraction necessary for the normal posture. On the other hand, it seems to be improbable, that a characteristic of the elasticity could have such a strong break in the slope, as the characteristic of the prothorax has when going through zero. Instead of this one might suppose a smoother form of the characteristic. Although because of this reason one cannot exclude this possibility, it seems to be more probable, that the animal uses a closed loop control system. This hypothesis is supported by the 
fact, that control systems with the required property of closed loop proportionality are described quantitatively or qualitatively for all leg joints (femurtibia-joint, coxa-trochanter-joint, thoracic-coxal-joint) in the stick insect (Bässler, 1965, 1967, 1972a, 1972b, 1973, 1974; Bässler et al., 1974; Wendler, 1964, 1972). Therefore the system controlling the height of a segment here assumed to be a feedback control system, could consist of these single and already known feedback systems in the different joints of the legs. But as a restriction one should remark, that the above experiments were done only in standing or fixed animals. Therefore it is not possible to say whether these closed loop control systems are also working in the free walking animal.

As in the abstract model discussed here the controllers of each segment, be they open or closed loop systems, work completely independently, of one another, no neuronal connections between the three controllers are required. This is possible, because the information transfer can be performed mechanically. This makes the model rather simple. Only the enlarged model discussed at the end of the Section $E$ requires a neuronal information channel between the pro- and the mesothoracical controller. Here the height of the prothorax has to be compared with that of the mesothorax and after that at least one of the three controllers has to be influenced by changing for instance its reference input.

In conclusion one can say, that the experimental results derived from free walking animals can be described within the exactness of measurement by a computer model. In addition to its rather simple structure, this model has the advantage, that it may be realized by putting together different subsystems which already are at least qualitatively known.

Acknowledgements: I wish to express my thanks to Dr. D. Graham for reading the English manuscript.

\section{References}

Bässler,U.: Proprioreceptoren am Subcoxal- und Femur-TibiaGelenk der Stabheuschrecke Carausius morosus und ihre Rolle bei der Wahrnehmung der Schwerkraftrichtung. Kybernetik 2, 168-193 (1965)

Bässler,U.: Zur Regelung der Stellung des Femur-Tibia-Gelenkes bei der Stabheuschrecke Carausius morosus in der Ruhe und im Lauf. Kybernetik 4, 18 -26 (1967)

Bässler,U.: Der „Kniesehnenreflex“ bei Carausius morosus: Übergangsfunktion und Frequenzgang. Kybernetik 11, 32-50 (1972a)

Bässler, U.: Der Regelkreis des Kniesehnenreflexes bei der Stabheuschrecke Carausius morosus: Reaktionen auf passive Bewegungen der Tibia. Kybernetik 12, 8-20 (1972b)

Bässler,U.: Zur Steuerung aktiver Bewegungen des Femur-TibiaGelenkes der Stabheuschrecke Carausius morosus. Kybernetik 13, 38-53 (1973)

Bässler, U.: Vom femoralen Chordotonalorgan gesteuerte Reaktionen bei der Stabheuschrecke Carausius morosus: Messung der von der Tibia erzeugten Kraft im aktiven und inaktiven Tier. Kybernetik 16, 213-226 (1974)

Bässler, U.: Zur Definition von Pro- und Metageotaxis bei Insekten. Biol. Cybernetics 19, 55-60 (1975)

Bässler, U., Cruse, H., Pflüger, H. J.: Der Regelkreis des Kniesehnenreflexes bei der Stabheuschrecke Carausius morosus: Untersuchungen zur Stabilität des Systems im inaktiven Tier. Kybernetik 15, 117-125 (1974)

Okhotsimskiy,D.Ye., Platonov,A.K., Borovin, G.K., Karpov, I.I.: Digital computer simulation of the motion of a stepping vehicle. Eng. Cybernetics 10, 410-419(1972)

Wendler, G.: Laufen und Stehen der Stabheuschrecke Carausius morosus: Sinnesborstenfelder in den Beingelenken als Glieder von Regelkreisen. Z. vergl. Physiol. 48, 198-250 (1964)

Wendler, G.: Körperhaltung bei der Stabheuschrecke: ihre Beziehungen zur Schwereorientierung und Mechanismen ihrer Regelung. Verh. Dtsch. Zool. Ges. 65, 214-218 (1972)

Wilson, D.M.: Insect walking. Ann. Rev. Entomol. 2, 103-122 (1966)

Received: April 1, 1976

Dr. H. Cruse

Fachbereich Biologie der Universität

Postfach 3049

D-6750 Kaiserslautern

Federal Republic of Germany 